\title{
Sampling in Precision IPM: When the Objective Is a Map
}

\author{
S. J. Fleischer, P. E. Blom, and R. Weisz
}

First and second authors: Department of Entomology, Pennsylvania State University, University Park 16802; and third author: Department of Crop Science, North Carolina State University, Raleigh 27695.

Accepted for publication 12 July 1999.

\begin{abstract}
Fleischer, S. J., Blom, P. E., and Weisz, R. 1999. Sampling in precision IPM: When the objective is a map. Phytopathology 89:1112-1118.

Measuring and understanding spatial variation of pests is a fundamental component of population dynamics. The resulting maps can drive spatially variable pest management, which we define as precision integrated pest management (IPM). Precision IPM has the potential to reduce insecticide use and slow the rate of resistance development because of the creation of temporally dynamic refuges. This approach to IPM requires sampling in which the objective is to measure spatial variation and map pest density or pressure. Interpolation of spatially referenced data is reviewed,
\end{abstract}

ABSTRACT and the influence of sampling design is suggested to be critical to the mapped visualization. Spatial sampling created problems with poor precision and small sample sizes that were partially alleviated with choosing sampling units based on their geostatistical properties, adopting global positioning system technology, and mapping local means. Mapping the probability of exceeding a threshold with indicator kriging is discussed as a decision-making tool for precision IPM. The different types of sampling patterns to deploy are discussed relative to the pest mapping objective.

Additional keywords: geostatistics, precision agriculture.
The early graphical development of the economic threshold (Fig. 4 in literature citation $12 ; 28$ ) conceptualizes a series of pest density maps marching through time. The entire map is above or below a general equilibrium density at different points in time, but at all points in time, parts of the population are shaded as above, and other parts below, an economic threshold. To facilitate integrated pest management (IPM), we simplify the map into a flat plane estimated by the mean and we sample to categorize the mean relative to a threshold or management criterion. This approach has been used to determine when, and when not, to apply management inputs. In other words, it has worked to help manage temporal variation.

Today, we find ourselves in a world focused on environmental issues in which geospatial information technologies are becoming increasingly accessible. When applied to agriculture, these technologies create the opportunity for management of both temporal and spatial variation (19). Allen et al. (1) conjectured what scouting might look like in the future if we were to apply a wide array of new technologies. Many of these technologies exist today, are rapidly advancing, and could be used to map pest populations within fields. We can capture greater biological realism by recognizing that populations vary in space, but this requires that the objective of the sampling be redefined as mapping the population rather than as estimating or categorizing the mean.

In this discussion, we focus on point-based sampling within agricultural fields. We recognize that the scale of the map should depend upon the biological organism being mapped, the agricultural or forested ecosystem being considered, and the intended use of the map. In entomology, there are more and further developed examples of mapping at a landscape or regional scale in forested or rangeland ecosystems (15) than in production agriculture, and the logical and technological tie to area-wide pest management pro-

Corresponding author: S. J. Fleischer; E-mail address: sjf4@psu.edu

Publication no. P-1999-0922-020

(C) 1999 The American Phytopathological Society grams are self-evident. We also recognize that pest maps come from sources other than point-based samples. Brewster and colleagues $(3,4)$ derived insect resource maps to drive population dynamic models from satellite imagery, and disease models are often driven by weather data. We hope that some of the concepts for pointbased sampling within agricultural fields are common to multiple scales and ecosystems. In any case, we should recognize that as infield and area-wide pest management progress to include mapping, we will see a convergence of spatial realism at several different scales. Quite simply, the technology will lead us in that direction. Setting the mapping of pest populations as a priority will accelerate the capture, analysis, and display of digital data, enabling collation of pest maps at multiple scales.

Mapping pests influences both our understanding and management of organisms. One objective of a recent book on the status of precision agriculture (23) was to understand the spatial variability of relevant variables and the processes that drive this variability at the within-field scale $(8,9)$. Mapping is improving our understanding of the spatial interactions between biological control agents and prey within grain storage bins (2) and commercial poultry houses $(30,31)$, which may lead to targeted approaches for inundative release of biological control agents. It is important that we not lose sight of this objective of understanding population dynamics as the technology advances.

Another objective of a map-based approach to IPM, or precision IPM, is reduced pesticide input. In replicated trials in potato fields, precision IPM was directly compared with 'conventional' IPM (in which sprays were applied to entire fields when means exceeded thresholds). Precision IPM reduced insecticides targeted against the Colorado potato beetle by 30 to $40 \%$ across a range of colonization pressures and also reduced pesticides targeted against the green peach aphid (36). However, lack of spatial stability and low thresholds in potatoes prevented insecticide reduction against potato leafhopper. Precision IPM in an urban and public health setting, as well as grain storage, also is being advanced by targeting prespecified percentages of the total population (2). This was effective in 
the chief petty officers' club of a submarine tender (a ship), for fumigation of stored grain pests, and in a mass rearing facility for a sterile male release program (2). Pesticide reduction on the submarine tender reached $99.7 \%$ of the surface area, compared with a traditional crack-and-crevice residual spray, while achieving a $90 \%$ pest population reduction.

Precision management affects more than pesticide load. It also changes the spatial distribution of the pesticide, resulting in unsprayed areas within the field. These unsprayed refuges often change from one date to the next, resulting in temporally dynamic refuges. The combination of reduced pesticide load and the creation of temporally dynamic refuges slowed the development of pesticide resistance and conserved natural enemies (16). This is an important finding, coming at a time when pesticide resistance has been increasing exponentially for decades and issues of how to manage resistance is a top priority to both entomologists and plant pathologists. However, the idea of precision IPM as a tool for resistance management should come as no surprise, since the concept of within-field refuges for susceptible phenotypes and natural enemies is often advanced as a management tool for resistance and conservation of biological control agents.

If we had pest maps, we could automate the targeting of management inputs. Technologies for variable rate application of all agricultural products, including pesticides, are being developed, and variable rate applications of fertilizers and lime are well into the early adoption stage in parts of the United States. Meanwhile, a wide range of other relevant variables in agriculture production (such as yield, quality and moisture of harvested product, soil fertility and physical parameters, and crop development and growth) are being mapped and used to influence the spatial (and temporal) deployment of management. Enabling technologies are rapidly becoming accessible, including the automation of positioning at very fine scales with global positioning systems and the management of spatially referenced data with geographic information systems. Because pests exhibit spatial variation, it makes sense to consider spatially varying management, but this will not occur until we are able to produce the pest or pest management maps. Currently, the difficulty of mapping pests and the speed of change in these maps has led the National Research Council (19) to question the feasibility of precision agriculture applied to pests other than weeds. It is entirely reasonable to suspect that there will be pest/crop and economic situations in which temporal management will be much more important than spatial management. However, there will also be situations in which spatial management will be valuable. If entomologists and plant pathologists are unable to produce the relevant maps, precision agriculture will proceed for virtually all aspects of plant agricultural production except insects and diseases.

In precision agriculture, we see a trend toward development of sensors. The sensor technology then becomes coupled with automation, including automation of positioning. Sampling questions about point estimates obtained with manual labor will become replaced with sampling questions associated with machines obtaining much larger quantities of data at much finer spatial resolutions (22). Development of sensor technology is essential for progress in precision IPM. With some significant exceptions $(3,4)$, however, IPM currently relies primarily on point-based sampling obtained with manual labor. This paper points to research topics for sampling when the objective is to make a map (some of which will carry over into machine-based sampling) and suggests that there is a potential for significant gains in IPM if and when we map pest density or damage. When the objective of a sampling effort is to make a map, the sampling unit, sample size, sample placement, spatial continuity of samples, and mapped visualization all influence each other.

\section{INTERPOLATION}

If we assume that spatially or georeferenced samples exist, we can begin by considering how to visualize the data and interpolate a surface model. Interpolation is the mathematical estimation of values at unsampled locations. It is analogous to extrapolation of a regression model, in that data are not present, but nearby data are used to create estimates. Scientists are often reluctant to extrapolate beyond their data, and those well-learned concerns are equally valid for interpolation. If you have serious reservations about the validity of nearby data with respect to their ability to provide reasonable estimates at unsampled locations, it may be wise to not interpolate a surface model. In that case, the data can be represented as a (spatially homogeneous) flat plane using a mean or other population measure (e.g., mode or median). However, if you wish to preserve the spatial information, maps can be produced without interpolation. Examples include posting values to the sampled locations (a posting map), displaying the posted values as a symbol (classified posting map), or displaying a circle in which the diameter is proportional to the observed value (buffered posting map). Roberts et al. (24) show insect density maps without interpolation used in IPM programs. These posting maps (or other maps) can be used to make management decisions or to direct further sampling.

Interpolation assumes that nearby samples provide information about unsampled locations. Maps (surface models) are created by estimating interpolated values at many unsampled locations, often in a grid of fine resolution, and displaying the gridded estimates. Wollenhaupt et al. (37) provide a concise review of interpolators used with point estimates of soil data, and that review is recommended for a fuller description and some historical and practical perspectives. In their treatment, global measures of central tendency (such as a mean or median) provide one type of interpolation. Moving averages estimate central tendencies from user-defined cells or windows, which can be overlapping, and these central tendencies become the interpolated values. Voronoi (or Thiessen) polygons construct areas around each sampled point that minimize the distance from unsampled locations to the sampled point. Values within that polygon are assigned from the sampled point within that polygon. Connecting the observed data points directly, a process called Delaunay triangulation, results in a set of triangles in which the longest side of each triangle is as short as possible. Interpolated values within the triangles become a linear function of the vertices of the triangle. Roberts et al. (24) show examples with insect data.

Several interpolation methods use the distance of observed samples to the unsampled location to help estimate the unknown value. Inverse-distance interpolators assign weights to nearby points based on their distance to the unsampled location, and the unsampled location is estimated as a weighted mean. However, these interpolators ignore or misspecify the spatial covariance function between sampled points. Weights are typically defined as an exponentially decreasing function of distance, with the exponent controlling how fast the weight decreases. The lower the exponent, the slower the decline in weight and the greater the influence of more distant points. Thus, lower exponents tend toward smoother maps, higher exponents tend toward preserving peaks of high values, and a zero exponent would reproduce a moving average map. Exponents of 1 to 3 are common with soil data (37), and exponents ranging from 2 to 6 have been used with insect data $(24,35)$.

Another interpolation technique that creates a weighted linear estimate of nearby points is kriging. In contrast to methods that assume some specific relationship with distance, kriging uses a statistical model of the degree of relatedness of pairs of points as a function of their intervening distance to define the weighting algorithm for use in kriging. The process of modeling spatial continuity is distinct from kriging, which uses the model for interpolation. The statistical model of spatial continuity defined from the data, called the variogram, has utility for aspects of sampling design and for understanding population dynamics beyond and in addition to its use for kriging. Both the analysis of spatial continuity and the interpolation with kriging are branches of geostatistics, and the reader is directed to the work by Isaaks and Srivastava (13) for a thorough applied treatment of this subject. Wollenhaupt et al. (37) 
also provide a very useful, concise review with respect to application for point sampling of soil data in precision agriculture.

There are several types of kriging. Point kriging works from the points, block kriging is an interpolation of areas, and indicator kriging is a categorical approach. Weights developed in point kriging minimize the average squared prediction error, and thus have been called the 'best' of the linear unbiased predictors (37). Also, because kriging is based upon a statistical model (in contrast to an assumed model in spline or inverse-distance methods), variances of the predicted values can be constructed. Models of spatial continuity and kriging interpolators can also consider direction as well as distance. When there is no directional bias, the model is called isotropic, otherwise it is considered anisotropic. Anisotropy was present in beetle density in potato fields (36) and in house fly density in manure piles (30), with spatial continuity at greater distances down, as opposed to across, crop or manure rows. Directional knowledge can be used to influence the search strategy for points to include in any of the weighted interpolators.

Two additional interpolations are useful. Splines are computationally intensive interpolators that iteratively balance goodnessof-fit and smoothness. When the variogram is used in the calculation of splines, this is equivalent to kriging (37). Trend surfaces are least squares linear regressions of the data to the $\mathrm{x}$ and $\mathrm{y}$ spatial coordinates, which can be calculated in many statistical software packages. Calculations could also readily include variables other than the coordinates, as in any multiple regression equation. Trend surfaces give a smooth view over the entire area, are useful for expression of spatial variation at a field scale, and may well provide most of the explanation of spatial variation of some pest populations (31). They are also useful for defining a spatial pattern that exists over the study area prior to examining for a smaller scale spatial pattern. For example, Sharov et al. (27) worked with an invading population across a large region, from well-established to newly invading populations. To model spatial variation at a finer scale, they first subtracted the trend and then conducted geostatistical analysis on the residuals from the trend surface model.

Interpolators that are combinations of nearby samples must first determine which points to include, which is done with a search strategy. Unconstrained search strategies are computationally expensive. Search strategies can be defined by distance, varied with direction (they could be ellipses aligned with crop rows) or constrained to ensure inclusion of points from all directions. This latter consideration is important when working with clustered samples or trends to ensure that estimates are not all derived from points in nearby clusters and to prevent bias.

Wollenhaupt et al. (37) reviewed literature comparing accuracy of different interpolators. They concluded that there was no single interpolator that was most accurate for all data. The main considerations, ranked in order of importance, were (i) sample strategy (sample placement and number of samples), (ii) the statistical properties of the sampled data, and (iii) the search strategy. Brenner et al. (2) argued for interpolation that came closest to reproducing trap counts at sampled locations ('exact' interpolators) over those that smoothed over nearby estimates ('smoothing' interpolators) by forcing a zero intercept into variogram models used for kriging, regardless of how well the data fit the variogram model. Weisz et al. (35) compared beetle counts and management decisions using splines, kriging, and inverse distance with three different exponents. Their analysis was restricted to data with sampling units and sampling spatial patterns that had provided the best models of spatial continuity in previous studies (34). Given this sampling, the choice of interpolator had little influence upon management decision or map error. All interpolators tended to have less error as mean density for the field increased. Gotway et al. (10) compared interpolators and sample spacing for their influence upon map accuracy and estimation of land areas of differing nitrate concentration from soil cores. Choice of interpolator had less influence than did sample spacing.

\section{PRECISION AND SPATIAL VARIATION OF THE SAMPLING UNIT}

The statistical properties of the sampled data are influenced by the sampling unit (the unit basis of area, plant part, time of trapping, etc., used to collect the samples). For interpolative mapping, sampling units ideally would exhibit reasonably strong spatial continuity over distances that exceed the distance between sample placement. However, the ability to resolve spatial dependence is compromised when sampling units have poor precision. Precision, which expresses the repeatability of a sample estimate, increases with sample size. In whole-field IPM, multiple sampling units are used to estimate a mean, and increasing sample size is one method of increasing precision of the estimated mean. When mapping, one or a few sampling units will be measured at each location. These few sampling units will be used for posting to a local site, interpolation, or modeling spatial continuity. Using a single (or a few) sampling unit(s) at each site can result in a local estimate with poor precision. Poor precision contributes to an inability to resolve spatial dependence and can result in maps in which the estimated values do not reproduce the observed values very well (34). Clusters of sampling units and mapping of local means may help solve this problem. Thus, Weisz et al. (34) developed sampling units that pooled over multiple individual stem counts for modeling spatial continuity and mapping, and Wollenhaupt et al. (37) recommend pooling multiple soil cores for each sampling location to decrease the coefficient of variation.

Pooling of sampling units to increase precision at the sampled site must be balanced against the reduction in the number and distribution of sample locations. Agriculture now has increasing access to technology for georeferencing of sample sites with the global positioning system (GPS) (33). Backpack GPS units can be adapted for use in IPM scouting, which enables each sampling unit, or composites of nearby sampling units, to be georeferenced. Current yield mapping technology creates streams of data, and the statistical properties of continuous georeferenced streams of samples for pest scouting has not yet been well documented. Block kriging, moving averages, or other interpolation tools that consolidate samples may also have applications in increasing precision and accuracy of maps.

Traditionally, discrete frequency distribution models, or indices of dispersion, have been used to infer spatial patterns $(5,6)$. At best, these models do this indirectly. Samples from a clumped spatial distribution tend to follow a negative binomial frequency distribution, while samples taken from a random spatial distribution tend not to deviate from a Poisson distribution. However, very different spatial patterns result in the same dispersion indices and discrete frequency distribution models. An example is a group of samples drawn from an aggregated population in which the aggregations are randomly distributed versus the same aggregations placed in a cline from one side to the other side of the field (17). These are different spatial patterns with the same dispersion indices and frequency distribution models.

For mapping, a more useful model of spatial variation considers the degree of relatedness of points as a function of their intervening distance and direction. A review of this geostatistical approach is beyond the scope of this paper, and the reader is directed to the work by Isaaks and Srivastava (13), Wollenhaupt et al. (37), and references therein. Publications using insect density include work by Schotzko and O'Keeffe (26), Rossi et al. (25), Leibhold et al. (15), Weisz et al. (34), and Sharov et al. (27). The traditional geostatistical tool for analyzing spatial dependence is the variogram (13). The variogram, however, assumes that local means and variances are stable across the region to be mapped. This is rarely true in insect populations, in which the variance tends to increase with the mean. Additional tools for spatial analysis of ecological data include the covariogram, which attempts to filter out variation in local means, and the correlogram, which attempts to filter out changes in variances (25). The correlogram tends to result in models with 
more definable characteristics when working with insect density $(25,34)$ and, thus, is more useful for kriging.

Variograms may be relatively flat, show a linear increase with distance, or reflect an asymptotic increase with respect to distance. Flat variograms suggest that the variance among pairs of points are as similar for points separated by small distances as for those separated by larger distances, i.e., the data exhibit no spatial autocorrelation. This can be due to the absence of variation that can be attributed to distance or due to an inability to resolve this variation. Spatial variation may be present, but not measured, in a variogram if the sampling units have poor precision or when samples are placed farther apart than the distances at which spatial variation occurs. Linearly increasing variograms, regardless of whether they are semivariograms, covariograms, or correlograms, result when the pattern is predominately a trend. In this case, variance among pairs of points tends to continuously increase with increasing distance (and trend modeling helps describe spatial variation). When pairs of points are more similar (less variable) when they are close together, and at some distance there is no further change in variance as distance increases, the variograms show an asymptotic increase to an upper 'sill.' This results in variograms with three parameters describing the spatial variability. The first is the nugget, which is either estimated as the y intercept (a modeled nugget) or measured as the value of the variogram at the shortest measured distance (an experimental nugget). As in a flat variogram, a nonzero nugget reflects spatial variability below the minimum distance between samples, poor precision, or both. Pooling sampling units can reduce the nugget if it is due to problems with precision. The sill is the upper asymptote of the variogram, which roughly corresponds to the population variance, but can be different due to erratic values or outliers. The range is the distance at which the variogram approximates the sill. This defines the estimated distance within which the samples remain spatially correlated.

Variograms of insect density are dynamic (8). They change with respect to the population process (colonization or in-field reproduction) that is predominant in the field at that time and may reflect more than one process simultaneously. They also change with respect to population phenology (the proportion of the population in a given life stage being sampled), population density, and spatial variation in the application of insecticides.

\section{PROBABILITY THRESHOLD MAPS}

It is often easier and faster to determine pest presence or absence, as opposed to enumerating pest density or damage, per sampling unit. Determining pest (or symptom) presence or absence in a sampling unit is a binomial form of categorical sampling. Categorical sampling need not be limited to presence/absence; more than one delineating value (often termed tally threshold) can be defined. Categorical sampling is more likely to be implemented (32), and sequential sampling plans have been built based on categorical sampling $(14,20)$. When sampling to create a map, the number of sampling units increases and, thus, the speed of using categorical sampling for mapping could be quite valuable. In the mapping literature, this has been termed indicator mapping.

The mining engineering literature (11) describe indicator mapping for determining when the benefits of developing a well at an unobserved site exceed the cost of extracting natural gas from that well based on categorical analysis of nearby observed well holes. The return from each observed well is categorized as cost effective or not (that is, the return exceeds the cost of drilling and developing the well). This results in a mapped set of $0 \mathrm{~s}$ and $1 \mathrm{~s}$, in which each observed well (point sample) has been categorized as below or above a threshold. The spatial continuity of the $0 \mathrm{~s}$ and $1 \mathrm{~s}$ is modeled with variography, and the resulting model is used to make a kriged interpolation surface. The resulting map shows values ranging from 0 to 1 expressing the probability of well holes at unobserved locations exceeding a threshold. Isaaks and Srivastiva (13) also provide examples of indicator mapping.

This approach of making probability threshold maps from categorical data may be useful for decision making in precision IPM. Brenner et al. (2) used the cumulative trap catch to define a threshold that included $85 \%$ of this function and then used indicator kriging to estimate the location and area of $85 \%$ of the sampled population. (Rather than model the variogram, Brenner et al. [2] forced variograms through a zero intercept. They felt this resulted in interpolated surface models that identified distinct 'hot spots,' or population foci, for targeted application.) An example of a map expressing the probability of Colorado potato beetle adults plus large larvae exceeding a threshold population density in potatoes when the mean field density was only slightly above threshold is shown in Figure 1. The samples (captured from 393 row-meter sampling units) are posted as closed circles when they exceed threshold and as open circles when below threshold. The observed samples were categorized as 0 (below threshold) or 1 (above threshold). The spatial continuity of the $0 \mathrm{~s}$ and $1 \mathrm{~s}$ were modeled with variography, and a correlogram was used to make a kriged interpolated surface, which estimates the probability of being above threshold (the probability of being scored as a 1) at any location.

A decision-making graphic using this probability threshold map to help decide at what probability contour to introduce controls is developed in Figure 2. First, two error rates (missed sprays and unnecessary sprays) were estimated by overlaying observed samples onto the mapped probability estimates of exceeding threshold (e.g., onto the map in Fig. 1). The proportion of missed spray sites was estimated by counting the number of observed sites that were above threshold but estimated as below threshold in the interpolated map and dividing that number by the total number of samples. The proportion of unnecessary spray sites was estimated by counting the number of observed sites that were below threshold but estimated as above threshold in the interpolated map and dividing that number by the total number of samples. These error rates were calculated while assuming that a spray was applied to the area at and above a given probability contour in the map in Figure 1; therefore, we obtain a unique error estimate for each map probability contour. For the entire map, the unnecessary control error rate is obtained when sprays are applied at the contour of 0 (at all areas in the map $\geq 0$ ), and the missed control error rate is obtained when sprays are applied at the contour of 1 (at all areas $\geq 1$; when no spray is applied). Concurrently, we estimated the amount of land area that would be sprayed at each map probability contour. The decision-making graphic (Fig. 2) displays the probability of the two types of error and the amount of land area associated with applying a spray at any given probability level. Conceptually, the process would use georeferenced categorical sampling to map the probability of exceeding a threshold and then use a graph expressing the rates of error and land area to help a grower decide at which probability contour to spray. It is then a simple process to turn the probability map into a management intervention map to define where to spray, release biological control agents, or apply other actions.

\section{SAMPLE PLACEMENT}

An interesting contrast between sampling program design in whole-field versus precision IPM is the relative emphasis placed on issues of sample size versus sample placement. In whole-field IPM, sampling programs are often designed to minimize the number of samples, often using sequential sampling designs. This is done to minimize in-field sampling labor (although practitioners note that travel time can be more expensive than in-field sampling time when using sequential sampling). Spatial placement is not considered in any analytical sense, except perhaps to suggest that an ' $\mathrm{X}$ ' or ' $\mathrm{O}$ ' is used to ensure that samples are collected from the entire field. There may be an implicit suggestion to avoid edges or other parts of a field that may harbor densities that are not repre- 
sentative of the entire field to ensure that the mean is unbiased. There may also be some admonition to try and place samples randomly, again to avoid bias. A more realistic view would recognize that it is unlikely that any sampling unit (such as one in the northwest corner of a field) has an equal probability of selection when the previous sample is taken from the southwest corner of a 20-acre field, which implies that sample placement is not truly random.

Sampling in precision agriculture, in contrast, places much more emphasis on where to take samples and provides the technology to follow through. Sample placement may be defined and georeferenced prior to entering the field and GPS equipment then used to guide the individual to the sampling site. In contrast to whole-field IPM, areas that have been avoided to guard against bias in the estimation of a field mean (e.g., field edges) may now become areas to delineate for variable management. Thus, in whole-field IPM, areas with distinctly high or low densities relative to a field mean have been a cause of concern, and avoided, but in site-specific IPM, it is precisely these areas that need to be delineated. A corollary to this emphasis in placement instead of sample size is the acceptance of a requirement for more samples. Implicit is the assumption that there is greater value in a map than a mean and, thus, there is an acceptance of greater sampling cost.

Wollenhaupt et al. (37) consider eight types of sample placement designs of soil cores in precision agriculture: judgmental sampling, simple random sampling, stratified random sampling, several types of cluster patterns, systematic patterns, stratified systematic unaligned sampling, search sampling, and use of auxiliary data layers. Judgmental sampling is when one relies upon one's (presumed good) judgment for sample placement, and Wollenhaupt et al. (37) suggest that bias almost always results. However, 'ranked set' sampling has been considered as a way to include expert opinion into a sampling pattern to achieve greater efficiency in estimating a mean (29). In ranked set sampling, one might ask a grower where he expects to find the highest and the lowest samples and base a design upon that information.

The basis for evaluating different designs for sample placement depends upon the objective and the evaluation criteria. Olea (21) considers sampling pattern optimization based on two criteria: the average standard error and the maximum standard error of predicted values at unsampled locations. When sampling from an underlying isotropic spatial distribution (e.g., no directional bias), he concludes that a regular hexagonal pattern best met his criteria. Sample patterns met his criteria in the following decreasing order: regular, stratified, random, and clustered. Thompson (29) notes that when sampling in a setting where spatial pattern exists, the objective may be to estimate a population quantity (a mean) for the entire area, predict a value at an unsampled site (the interpolation problem), or find (delineate) areas of importance (such as high values). He suggests that systematic or stratified designs are often efficient for the first two objectives. The variance of population quantities (such as a total or mean) that are formed as a linear combination of the data is a linear combination of embedded covariances contained within the data set. Thus, it becomes efficient (you tend to minimize the variance of the population quantity) by minimizing the covariates (29). This is often accomplished by placing samples as far apart as possible, which systematic or stratified designs that

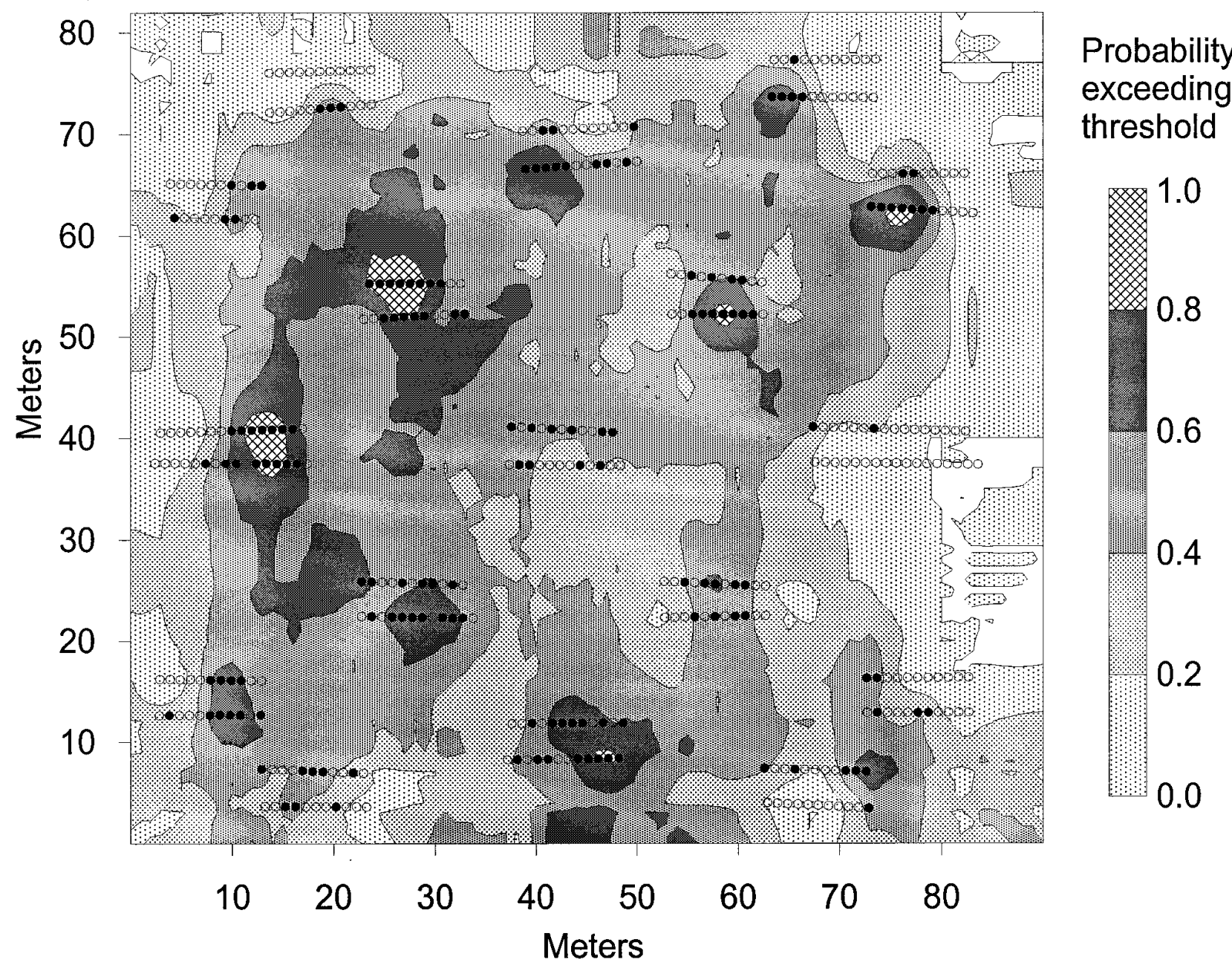

Fig. 1. Map expressing the probability of Colorado potato beetle adults plus large larvae exceeding a threshold 8 weeks following planting. x,y Units are in meters, and $z$ is the probability of exceeding a threshold estimated with indicator kriging. Mean density for the field slightly exceeded the threshold. Sample locations $(n=393)$ are posted as above (closed circle) or below (open circle) the threshold. 
arrange samples over the entire field tend to do. Midgarden et al. (17) recognize spatial variation in insect trap counts, yet their sampling objective is to estimate a mean for a field. Therefore, they use their models of spatial continuity to define a range of spatial dependence and suggested placing samples beyond this range to achieve an unbiased mean. Sampling beyond the estimated range of spatial dependence would tend to minimize the spatial covariance contribution to the variance of a mean.

When interpolated estimates are needed, samples are ideally placed within the range of spatial dependence to allow the information inherent in the spatial covariance to contribute, via weighted estimates, to the interpolated value. Systematic and stratified designs can also be efficient for sample placement when the data will be used for interpolation and when the evaluation criteria is to minimize the largest mean square prediction error from interpolated values $(21,29)$. With this evaluation criterion, it is valuable to minimize the distance between any location to be interpolated and a sampled location. Systematic or stratified designs tend to help because they minimize the maximum distance between sampled and unsampled sites. Thompson (29) and Wollenhaupt et al. (37), however, caution that systematic patterns can produce very biased designs when the periodicity of the sampling locations in a systematic pattern coincide with the periodicity of another variable. In agricultural systems, periodicity is anthropogenic and creates locations that are easier to sample (e.g., spray rows, drain tiles, etc.). Wollenhaupt et al. (37) suggest that periodic patterns are often present in agroecosystems because of historical management and recommend unaligned grids to ensure against this type of bias. Unaligned grids are sampling patterns that add a local degree of randomness to systematic grids. In marked contrast to the ideal of random sampling for an unbiased mean in whole-field IPM, Wollenhaupt et al. (37) and Thompson (29) discourage simple random sampling for mapping, because it can easily result in placement of some samples in clusters while leaving other areas unsampled.

Wollenhaupt et al. (37) make a clear distinction between stratified or systematic sampling and unaligned sampling. In all three patterns, the field is first divided (stratified) into cells (typically of equal area) and samples taken from within each cell. When the location within a cell is chosen at random, the pattern is termed stratified random. When the location within a cell is fixed for all cells (e.g., the center of the cell), the pattern is called systematic and is suggested to be somewhat more precise than stratified random. To avoid periodicity bias, an unaligned choice of locations within cells is recommended. To achieve this, the cell is further subdivided into subcells. A random (x,y) location is chosen in, for example, the southwest cell. The $\mathrm{x}$ location is then held constant in each cell to the east, and the y location is varied at random. In the next row to the north, a new x location is chosen at random without replacement, and the y location is held constant. The result is that the $\mathrm{x}$ locations are the same in the rows, the y directions are the same in the columns, and the samples are spaced approximately equally but are not aligned. In a geostatistical analysis of sample placement for mapping insect populations, Schotzko and O'Keeffe (26) find that a hexagonal grid results in better map precision than a uniform (systematic) grid. Those used to looking at honeybee hives will intuitively relate to the efficiency inherent in the hexagonal pattern of a honeycomb.

Random or various types of systematic patterns assume no knowledge about the field. However, the ranked set approach mentioned above is influenced by educated guesses about where to find areas of interest (high or low samples). Many other additional data layers are becoming increasingly available, including soil plant nutrient maps, remote sensing imagery, soil maps, yield maps, landscape position and elevation maps, etc. To the degree that the information in these maps correlates with the pest or damage being mapped, they could be incorporated into the sampling placement design as well as the interpolation algorithm. Mulla (18) uses covariography to develop one map layer from another, including the targeting of sampling locations. Brewster and Allen (3) use remotely sensed imagery to create host maps that they are able to correlate with whitefly density and, thus, use to create whitefly density maps as inputs into spatiotemporal dynamic models. Their sample patterns are defined by the agroecosystem landscape. Ellsbury et al. (7) show spatial covariation between corn rootworm emergence patterns and landscape position and suggest that landscape position information might be useful to allocate sampling effort or placement. Insects often show 'edge effects,' in which densities are distinctly higher or lower relative to field edges or related landscape features. These patterns could be quantified and used to allocate sample placement. This form of what is often termed 'targeted sampling' has special relevance to weekly scouting programs in IPM. In IPM scouting programs, the map from the previous week is an obvious potential knowledge base from which to place samples.

Information about the area to be mapped also occurs during the process of collecting samples. Thompson (29), and references therein, describe adaptive sampling, in which the objective is to gain efficiency by allocating relatively more sampling effort (by the placement of more samples) in areas of interest. Sequential sampling also uses incoming information to influence the sampling process by defining when to stop collecting what are presumed to be independent samples. Thus, sequential sampling strives to allocate more samples in fields when the whole-field population estimate is close to a value of interest. Sequential sampling can be viewed as a subset of adaptive sampling plans, designed for whole fields and with the assumption of spatial independence among samples.

Rather than assume spatial independence, adaptive sampling tries to capitalize on the spatial covariance of the data and addresses sample placement within fields rather than sample size for a whole field. Theoretically, adaptive sampling should increase efficiency whenever the evaluation criteria (such as minimizing an error term) varies among different choices of a second set of samples, conditional upon the first set of samples. Thompson (29) suggests that efficiency tends not to increase over a conventional (e.g., systematic) design with data that follow a Gaussian white noise or a Poisson process with no spatial covariance. However, efficiency is increased with log-Guassian or spatially correlated data. Thompson (29) describes two adaptive designs that may be useful for field settings. In both, initial samples are taken with a conventional design, which, for mapping, is often a systematic grid or something similar. In adaptive cluster sampling, neighboring sampling locations are added

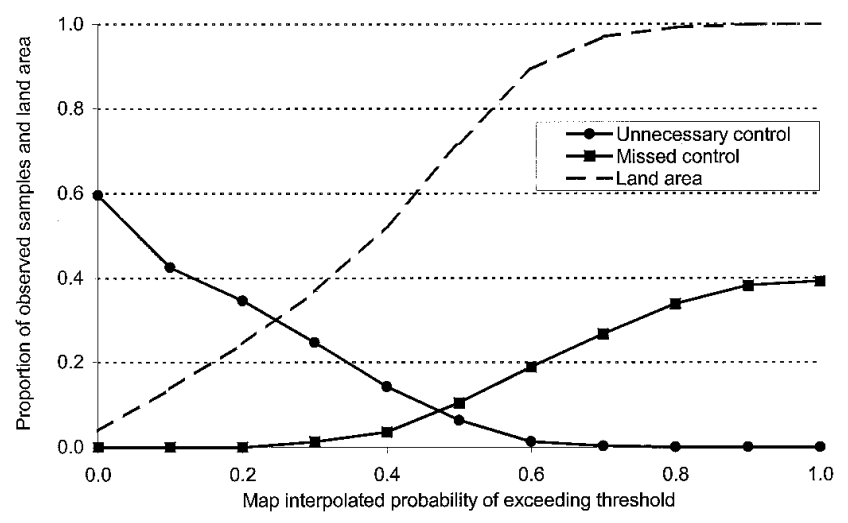

Fig. 2. Decision support graphic representing management error and land area associated with spraying the field in Figure 1 at any given probability contour and above (spraying the entire field is represented as spraying at a contour of 0 , which is spraying all area in the map at $\geq 0$ ). The $x$ axis refers to the probability contours shown in Figure 1. Two types of error are estimated for spraying at each contour: (i) unnecessary control (spraying observed samples that were below threshold), and (ii) missed control (not spraying observed samples that were above threshold). The land area curve shows the cumulative proportion of unsprayed land area at each probability contour. Growers could balance cost (land area treated) against error rates to select a probability threshold that delineates the spray map. 
whenever a prespecified value (e.g., a threshold) is observed. In adaptive allocation sampling, additional sampling is allocated based on the local variability (e.g., the local standard deviation) found in the initial sample set. Relative efficiency (the variance of the estimator with a conventional strategy divided by the variance obtained with an adaptive plan) of adaptive sampling programs increased in almost all studies compiled by Thompson (29) with point process data, including trees, and with organisms such as shrimp, fish, and birds. However, there were no examples provided with plant pathogens, plant stress symptoms, or insects. For IPM, it would be intriguing to consider the 'initial' sample to be that obtained in the sample set from week one, and try to use adaptive designs to optimize the sample placement in week two (or beyond).

\section{ACKNOWLEDGMENTS}

We thank G. Hughes for encouragement and his energy devoted to the symposia; P. Tobin and D. Midgarden, Ph.D. students at Penn State, for valuable comments on earlier drafts of this manuscript; and Z. Smilowitz for continued support in the mapping of insect density.

\section{LITERATURE CITED}

1. Allen, J., Kopp, D. D., Brewster, C. C., and Fleischer, S. J. 1999. 2011: An agricultural odyssey. Am. Entomol. 45:96-104.

2. Brenner, R. J., Focks, D. A., Arbogast, R. T., Weaver, D. K., and Shuman, D. 1998. Practical use of spatial analysis in precision targeting for integrated pest management. Am. Entomol. 44:79-101.

3. Brewster, C. C., and Allen, J. C. 1997. Spatiotemporal model for studying insect dynamics in large-scale cropping systems. Environ. Entomol. 26:473-482.

4. Brewster, C. C., Allen, J. C., and Kopp, D. D. 1999. IPM from space: Using satellite imagery to study regional crop-insect interactions. Am. Entomol. 45:105-117.

5. Davis, P. M. 1994. Statistics for describing populations. Pages 33-54 in: Handbook for Sampling Methods for Arthropods in Agriculture. L. P. Pedigo and G. D. Buntin, eds. CRC Press, Boca Raton, FL.

6. Elliott, J. M. 1977. Some methods for the statistical analysis of samples of benthic invertebrates, 2nd ed. Freshwater Biological Association, Scientific Publication 25. The Ferry House, Westmoreland, England.

7. Ellsbury, M. M., Woodson, W. D., Malo, D. D., Clay, D. E., Carlson, C. G., and Clay, S. A. 1998. Spatial variability in corn rootworm distribution in relation to spatially variable soil factors and crop condition. Pages 523-534 in: Proc. Int. Conf. Precision Agric., 4th. P. Robert, ed. American Society of Agronomy, Madison, WI.

8. Fleischer, S. J., Blom, P., Emmen, D., and Hower, A. 1998. Dynamics in the spatial continuity of insect density. Pages 133-141 in: Proc. Int. Conf. Precision Agric., 4th. P. Robert, ed. American Society of Agronomy, Madison, WI.

9. Fleischer, S. J., Weisz, R., Smilowitz, Z., and Midgarden, D. 1997. Spatial variation in insect populations and site-specific integrated pest management. Pages 101-130 in: The State of Site-Specific Management for Agriculture. F. J. Pierce and E. J. Sadler, eds. American Society of Agronomy, Crop Science Society of America, and Soil Science Society of America, Madison, WI.

10. Gotway, C. A., Ferguson, R. B., Hergert, G. W., and Peterson, T. A. 1996. Comparison of kriging and inverse-distance methods for mapping soil parameters. Soil Sci. Soc. Am. J. 60:1237-1247.

11. Hohn, M. E. 1988. Geostatistics and Petroleum Geology. Van Nostrand Reinhold, New York.

12. Hughes, G. 1999. Sampling for decision making in crop loss assessment and pest management: Introduction. Phytopathology 89:1080-1083.

13. Isaaks, E. H., and Srivastava, R. M. 1989. Applied Geostatistics. Oxford University Press, New York

14. Jones, V. P. 1994. Sequential estimation and classification procedures for binomial counts. Pages 175-205 in: Handbook for Sampling Methods for Arthropods in Agriculture. L. P. Pedigo and G. D. Buntin, eds. CRC Press, Boca Raton, FL.

15. Liebhold, A. M., Rossi, R. E., and Kemp, W. P. 1993. Geostatistics and geographic information systems in applied insect ecology. Annu. Rev. Entomol. 38:303-327.

16. Midgarden, D. M., Fleischer, S. J., Weisz, R., and Smilowitz, Z. 1997. Impact of site-specific IPM on the development of esfenvalerate resistance in Colorado potato beetle (Coleoptera: Chrysomelidae) and on population densities of natural enemies. J. Econ. Entomol. 90:855-867.

17. Midgarden, D. G., Youngman, R. R., and Fleischer, S. J. 1993. Spatial analysis of counts of western corn rootworm (Coleoptera: Chrysomelidae) adults on yellow sticky traps in corn: Geostatistics and dispersion indices. Environ. Entomol. 22:1124-1133.

18. Mulla, D. J. 1997. Geostatistics, remote sensing and precision farming. Pages 100-119 in: Ciba Foundation Symposium: Precision Agriculture: Spatial and Temporal Variability of Environmental Quality. J. Wiley \& Sons, Chichester, England.

19. National Research Council. 1997. Precision Agriculture in the 21st Century. Geospatial and Information Technologies in Crop Management. National Academy Press, Washington, DC.

20. Nyrop, J. P., Agnello, A. M., Kovach, J., and Reissig, W. H. 1989. Binomial sequential classification sampling plans for European red mite (Acari: Tetranychidae) with special reference to performance criteria. J. Econ. Entomol. 82:483-490.

21. Olea, R. A. 1984. Sampling design optimization for spatial functions. Math. Geol. 16:369-392.

22. Pierce, F. J., Anderson, N. W., Colvin, T. S., Schueller, J. K., Humburg, D. S., and McLaughlin, N. B. 1997. Yield mapping. Pages 211-244 in: The State of Site-Specific Management for Agriculture. F. J. Pierce and E. J. Sadler, eds. American Society of Agronomy, Crop Science Society of America, and Soil Science Society of America, Madison, WI.

23. Pierce, F. J., and Sadler, E. J., eds. 1997. The State of Site-Specific Management for Agriculture. American Society of Agronomy, Crop Science Society of America, and Soil Science Society of America, Madison, WI.

24. Roberts, E. A., Ravlin, F. W., and Fleischer, S. J. 1993. Spatial data representation for integrated pest management programs. Am. Entomol. 39:92-107.

25. Rossi, R. E., Mulla, D. J., Journel, A. G., and Franz, E. H. 1992. Geostatistical tools for modeling and interpreting ecological spatial dependence. Ecol. Monogr. 62:277-314.

26. Schotzko, D. J., and O'Keeffe, L. E. 1990. Effect of sample placement on the geostatistical analysis of the spatial distribution of Lygus hesperus (Heteroptera: Miridae) in lentils. Econ. Entomol. 83:1888-1900.

27. Sharov, A. A., Liebhold, A., and Roberts, E. A. 1996. Spatial variation among counts of gypsy moths (Lepidoptera: Lymantriidae) in pheromonebaited traps at expanding population fronts. Environ. Entomol. 25:13121320.

28. Stern, V. M., Smith, R. F., van den Bosch, R., and Hagen, K. 1959. The integrated control concept. Hilgardia 29:81-100.

29. Thompson, S. K. 1997. Spatial sampling. Pages 161-172 in: Ciba Foundation Symposium: Precision Agriculture: Spatial and Temporal Variability of Environmental Quality. J. Wiley \& Sons, Chichester, England.

30. Tobin, P. C. 1997. Spatio-temporal dynamics of the house fly, Musca domestica L. (Diptera: Muscidae), and the predator Carcinops pumilio (Erichson) (Coleoptera: Histeridae) in high-rise poultry facilities. M.S. thesis. Pennsylvania State University, University Park.

31. Tobin, P. C., Fleischer, S. J., and Pitts, C. W. 1999. Spatio-temporal dynamics of resident and immigrating populations of Carcinops pumilo (Erichson) (Coleoptera: Histeridae) in high-rise poultry facilities. J. Med. Entomol. 36:568-577.

32. Trumble, J. T. 1994. Sampling arthropod pests in vegetables. Pages 603626 in: Handbook for Sampling Methods for Arthropods in Agriculture. L. P. Pedigo and G. D. Buntin, eds. CRC Press, Boca Raton, FL.

33. Tyler, D. A., Roberts, D. W., and Nielsen, G. A. 1997. Location and guidance for site-specific agriculture. Pages 161-181 in: The State of SiteSpecific Management for Agriculture. F. J. Pierce and E. J. Sadler, eds. American Society of Agronomy, Crop Science Society of America, and Soil Science Society of America, Madison, WI.

34. Weisz, R., Fleischer, S. J., and Smilowitz, Z. 1995. Site-specific integrated pest management for high value crops: Sample units for map generation using the Colorado potato beetle (Coleoptera: Chrysomelidae) as a model system. J. Econ. Entomol. 88:1069-1080.

35. Weisz, R., Fleischer, S. J., and Smilowitz, Z. 1995. Map generation in high-value horticultural integrated pest management: Appropriate interpolation methods for site-specific pest management of the Colorado potato beetle (Coleoptera: Chrysomelidae). J. Econ. Entomol. 88:1650-1657.

36. Weisz, R., Fleischer, S. J., and Smilowitz, Z. 1996. Site-specific integrated pest management for high value crops: Impact on potato pest management. J. Econ. Entomol. 89:501-509.

37. Wollenhaupt, N. C., Mulla, D. J., and Gotway-Crawford, C. A. 1997. Soil sampling and interpolation techniques for mapping spatial variability of soil properties. Pages 19-53 in: The State of Site-Specific Management for Agriculture. F. J. Pierce and E. J. Sadler, eds. American Society of Agronomy, Crop Science Society of America, and Soil Science Society of America, Madison, WI. 